\title{
Feedback control of noise in spin valves by the spin-transfer torque
}

\author{
Swarnali Bandopadhyay, ${ }_{1}^{1}$ Arne Brataas, ${ }^{1}$ and Gerrit E. W. Bauer ${ }^{2}$ \\ ${ }^{1)}$ Department of Physics, Norwegian University of Science and Technology, 7491, Trondheim, \\ Norway \\ ${ }^{2)}$ Kavli Institute of NanoScience, Delft University of Technology, Lorentzweg 1, 2628 CJ Delft, \\ The Netherlands
}

(Dated: 26 November 2018)

The miniaturisation of magnetic read heads and random access memory elements makes them vulnerable to thermal fluctuations. We demonstrate how current-induced spin-transfer torques can be used to suppress the effects of thermal fluctuations. This enhances the fidelity of perpendicular magnetic spin valves. The simplest realization is a dc current to stabilize the free magnetic layers. The power can be significantly reduced without losing fidelity by simple control schemes, in which the stabilizing current-induced spin-transfer torque is controlled by the instantaneous resistance.

PACS numbers: 72.25.-b,76.50.+g,75.70.Cn,72.80.Ga,75.60.Jk

Magnetic spin valves with metal or insulator spacers are used for hard disk read heads as well as for magnetic random access memory elements. The physical principle of operation is the giant magnetoresistance (GMR) or tunnel magnetoresistance (TMR), which vary with the relative magnetization between a layer with fixed magnetization (e.g. by exchange biasing) and a free layer with a magnetization that is allowed to (re)orient the magnetization direction. Further miniaturization is thwarted by the increased thermal fluctuations associated with smaller magnets, however, because the magnetic anisotropy energies scale with the total magnetic moment 1 . GMR read heads in the form of nanometerscale pillars are currently considered as possible replacements for TMR read heads ${ }^{2}$. Their higher sensitivity comes at the cost of noise which is enhanced by the spintransfer-torque-induced coupling of electronic and magnetic fluctuations ${ }^{3,4}$. Here we demonstrate that the spintransfer torque can also be used to suppress the noise in spin valves. The necessary power to achieve a given fidelity can significantly be reduced by feedback control of the electric current-induced torque by the instantaneous resistance.

An electric current passed through a spin valve interacts with the magnetic order parameter when the magnetizations are non-collinear. This is caused by the spin current component polarized normal to the magnetization of the free layer, which is absorbed at the interface. The loss of angular momentum in the spin current is transferred to the magnetization as a spin-transfer torque,,-10 which effectively leads to magnetic damping or anti-damping of the magnetization dynamics, depending on the current direction. Low frequency noise can be suppressed by increasing the magnetic anisotropy of the free magnetic layer at the cost of reduced sensitivity. Covington ${ }^{3}$ proposes to reduce noise by applying a spintransfer torque which opposes the intrinsic damping so that the effective damping is reduced. According to the fluctuation-dissipation theorem (FDT) the random field that perturbs the magnetization at thermal equilibrium is indeed proportional to the Gilbert damping constant

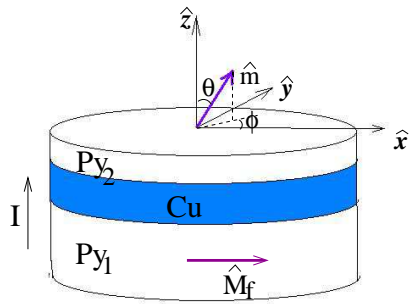

FIG. 1. (Colour online) Schematic diagram of a perpendicular spin valve under study. A non-magnetic spacer layer $(\mathrm{Cu})$ is sandwiched between a thick ferromagnetic $\left(\mathrm{Py}_{1}\right)$ layer with 'fixed' magnetization along $\hat{M}_{\mathrm{f}}$ and a thin ferromagnetic layer $\left(\mathrm{Py}_{2}\right)$ with magnetization $\hat{m}$ which is relatively 'free' to move. A positive electric charge current $\vec{I}$ is defined to flow from the 'fixed' layer to 'free' layer.

(see Eq. (3) below). A reduced damping therefore reduces noise. However, in the presence of a spin-transfer torque driving force the FDT does not hold and a reduced damping does not improve read-head performance. In this Letter we prove that the opposite is true: we have to increase the damping in order to suppress the noise. Applying a dc current of the right direction we are able to increase the fidelity of the read head compared to the zero current situation. The draw back of this approach is the additional power that has to be invested for a given noise suppression. In search of a more energy-conserving method we propose to apply a time-dependent current that is controlled by the instantaneous magnetization configuration as measured by the GMR/TMR. We implement here two physically transparent feedback mechanisms, noting that performance could even be improved by making full use of sophisticated control theory.

Our model corresponds to a realistic $\underline{11,12}$ nanopillar $(\mathrm{FM}|\mathrm{NM}| \mathrm{FM})$ spin-valve as sketched in Fig. 1, consisting of a thick ferromagnet (FM) permalloy (Py) layer with fixed magnetization direction $\hat{M}_{\mathrm{f}}$ and a thin Py layer separated by a non-magnetic $(\mathrm{Cu})$ layer. The pillar has an elliptical cross-section, which defines the easy axis of the 
free layer to be along $\hat{x}$. A charge current $I$ flowing from the thick layer to the thin layer in the perpendicular direction $(\hat{z})$ is defined to be positive. The device is stable for a dc current up to $10^{12} \mathrm{~A} / \mathrm{m}^{2} \underline{11}$. The allowed peak value for a pulsed current is higher 13 .

We assume a spatially uniform magnetization of the free FM layer ('macrospin' approximation). The temporal evolution of the magnetization vector $\hat{m}=\vec{M} / M_{s}$ of the free FM in presence of random field and a Slonczewski spin-transfer torque model can be described by the Landau-Lifshitz-Gilbert equation:

$$
\begin{aligned}
\frac{d \hat{m}}{d t}= & -\gamma \hat{m} \times \vec{H}_{\mathrm{eff}}+\alpha \hat{m} \times \frac{d \hat{m}}{d t} \\
& -\gamma \hat{m} \times \vec{h}(t)+\gamma a_{J} \hat{m} \times\left(\hat{m} \times \hat{M}_{\mathrm{f}}\right) .
\end{aligned}
$$

The effective field $\vec{H}_{\text {eff }}=-\partial E(\vec{M}) / \partial \vec{M}$ depends on the free energy density

$E(\vec{M})=-\frac{1}{2} H_{\mathrm{an}} M_{s}(\hat{m} \cdot \hat{e})^{2}-\vec{H}_{\mathrm{ext}} \cdot \vec{M}+2 \pi M_{s}^{2}(\hat{m} \cdot \hat{z})^{2}$,

where $\hat{e}$ is the unit vector along the easy-axis. $\left|\vec{H}_{\text {an }}\right|=$ $\left(N_{y}-N_{x}\right) M_{s}=0.05 \mathrm{k}$ Oe is the uniaxial anisotropic field and $\left(N_{z}-N_{y}\right) M_{s}=4 \pi M_{s}(=5 \mathrm{k} \mathrm{Oe}) \stackrel{11}{ }$ is the demagnetization field (last term in the free energy Eq. (2)) with demagnetization factors $N_{x}, N_{y}, N_{z} \underline{14}$ and $M_{s}$ is the saturation magnetization. A weak $\left(<0.38 \mathrm{k} \mathrm{Oe}{ }^{11}\right)$ external field $\left|\vec{H}_{\text {ext }}\right|=0.1 \mathrm{k}$ Oe is chosen to ensure no switching due to an applied in-plane field. The damping torque in Eq. (11) is proportional to the Gilbert constant $\alpha=0.01$. The next entry on the r.h.s. of Eq. (1) is a stochastic term. The stochastic field $\vec{h}(t)$ has zero mean and white noise correlation 15 .

$$
\left\langle h^{(i)}(t) h^{(j)}\left(t^{\prime}\right)\right\rangle=\frac{2 k_{B} T \alpha}{\gamma M_{s} V} \delta_{i j} \delta\left(t-t^{\prime}\right),
$$

where $k_{B} T$ is the thermal energy, $\gamma=0.176 \times 10^{8} \mathrm{G}^{-1} \mathrm{~S}^{-1}$ is the gyromagnetic ratio and $V$ the volume of the free ferromagnet, and $\langle\cdots\rangle$ denotes statistical averaging. The last term on the r.h.s. of Eq.(11) is the spin-transfer torque parameterized by $a_{J}$ which has the dimension of magnetic field and it is proportional to applied current $I$ as $a_{J}=(\hbar / 2 e) \eta\left(I / 4 \pi M_{s} V\right)$ where $\eta(=50 \%)$ is the spinpolarisation.

We solve the Landau-Lifshitz-Gilbert equation numerically in the presence of stochastic torques at room temperature by the Heun method $\underline{16}$. We use discretized time interval $d t=1 \mathrm{ps}$. We start from the initial state $m_{x}^{(0)}=1$ and study the temporal evolution of $m_{x}$. We calculate the fidelity life time, which is defined as the average time it takes until the magnetization deviates from its initial equilibrium value beyond a cutoff $m_{c}=0.8$ (arbitrary choice). The typical value of fidelity life time in absence of applied current $\tau_{0}$ is 500 ps as obtained from our calculations. We average over 10000 realizations.

Let us first study the dynamics in the presence of a dc current and compute the fidelity time enhancement

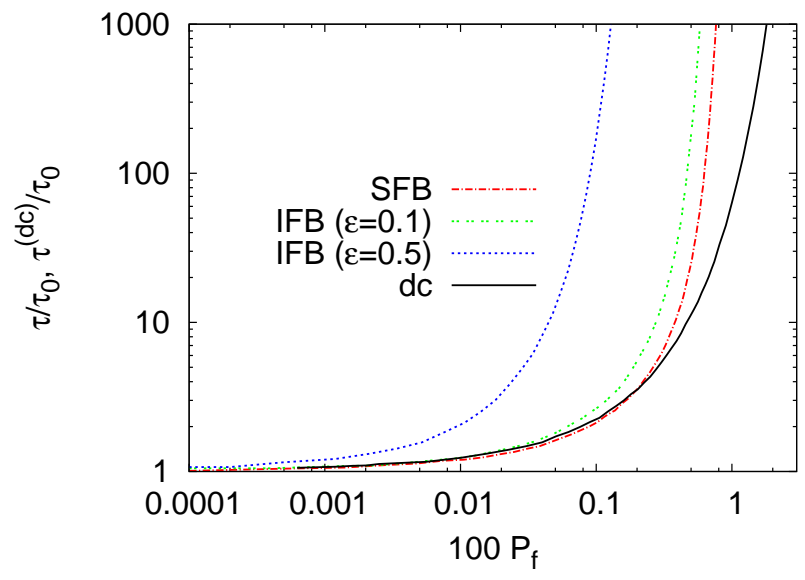

FIG. 2. (Colour online) Fidelity life-time $\tau$ in the presence of a dc current, as well as a pulsed current with constant magnitude (referred as simple feedback 'SFB') and varying magnitude (referred as improved feedback 'IFB'; larger $\epsilon$ indicates stronger variation) in units of $\tau_{0}$, the fidelity life time in the absence of a spin-torque, as a function of the dimensionless average power consumption factor $P_{f}$. Results are obtained at room temperature and averaged over 10000 realizations.

$\tau / \tau_{0}$ and the average dimensionless power consumption factor $P_{f}=\left(a_{J} / 4 \pi M_{s}\right)^{2}$ (the solid curve in Fig. 2). The dimensionless fidelity $\tau / \tau_{0}$ increases exponentially with the square of $P_{f}$. Thus fidelity increases with magnitude of applied current (hence torque). As a test of our method we repeated the simulations of current induced magnetization reversal of Ref $\underline{\underline{17}}$ and found good agreement.

We propose two control methods to reduce power consumption, assuming that we can monitor the instantaneous magnetization in real time by the electrical resistance. First, we let the magnetization evolve from its initial equilibrium configuration in the absence of a spin-torque and apply a current pulse with constant magnitude when $m_{x}$ drops below a chosen lower bound $m_{l}(=0.85)$. We switch off the current pulse once $m_{x}$ reaches a chosen upper bound $m_{u}(=0.95)$. We refer to this method as the simple feedback scheme (SFB). We compute the dimensionless fidelity life time $\tau / \tau_{0}$ and the dimensionless average power consumption factor $P_{f}=\left(a_{J} / 4 \pi M_{s}\right)^{2}(\Delta t / \tau)$, where $\Delta t$ is the total duration over which the spin-torque is in action. The result is shown as the dash-dotted line in Fig. 2, The fidelity life time as a function of invested power is very similar to the dc case. In the large power regime which also refers to large current regime, the SFB leads to significant power savings, however. A fidelity increased by a factor 1000 compared to the zero-torque situation can be gained by investing $\sim 3$ times less power as compared to the dc torque method. Here our approach is to apply a larger torque for shorter duration as large current ensures enhancement in fidelity and small duration makes 
the scheme power-saving one. Note that in our numerical simulation the magnitude of current pulses would be such that the magnetization switches slowly in the timescale of ps, the chosen time-step. In the small $P_{f}$ regime, the smaller enhancement in fidelity (compare the dashdotted curve with the solid one in Fig. (2) than dc case is due to the initial delay, set by $m_{l}$ on $m_{x}$. With increasing torque, the initial delay becomes insignificant.

At this stage we devise an improved control of the current pulses to save much energy. This case will be called as improved feedback method (IFB). As in the 'SFB' case, we allow the magnetization to evolve from its initial state without applying a current. When $m_{x}$ drops below $m_{l}$ we turn on a pulsed current corresponding to a torque, which now is a function of $m_{x}$. We have chosen $a_{J}=a_{J}^{(0)}\left[\left(m_{l}-m_{c}\right) /\left(m_{x}-m_{c}\right)\right]^{\epsilon}$, where $a_{J}^{(0)}$ corresponds to the initial value of the current pulse when it is turned on. $m_{l}$ and $m_{c}$ are two chosen cutoff values for $m_{x}$ as described above. Note that in the SFB case magnitude of the pulse current is $a_{J}=a_{J}^{(0)}$ whenever it is in action but in the present case $a_{J}$ increases monotonically when $m_{x}$ drops from $m_{l}$ and then decreases with increasing $m_{x}$ from $m_{c}$ in presence of spintorque. Thus to estimate invested power we accumulate the power consumption factor at each time step i.e. $P_{f}=\sum_{i=1}^{N}\left(a_{J_{i}} / 4 \pi M_{s}\right)^{2}\left(t_{i} / \tau\right)$. Since in the simulation we are using a finite time step, in practice, we do not reach the maximal current limit as $m_{x} \rightarrow m_{c}$. As soon as $m_{x}$ reaches $m_{u}$ we turn off the current again as in the SFB case. We carry out simulations for two values of the exponent $\epsilon$ in Fig. 2, For small $(\epsilon=0.1)$, there is only a slight improvement (the double dashed curve in Fig. 2) in the result as compared to the SFB case. For an intermediate value $(\epsilon=0.5)$, a significant improvement (the dashed curve in Fig. (2) is visible. A fidelity enhancement by a factor 1000 costs roughly 15 times less power than in the dc limit. Larger $\epsilon$ requires a smaller size of time-step to get reliable convergence in the numerical integration.

We now estimate the typical power consumption of our feedback loop in a practical situation. The average power consumption is $P=I^{2} R(\Delta t / \tau)=C_{\mathrm{S}} P_{f}$ with $C_{\mathrm{S}}=I_{\mathrm{S}}^{2} R$ and $I_{\mathrm{S}}=(2 e V / \hbar \eta) 4 \pi M_{s}^{2}$ are system specific constant terms. For our system $I_{\mathrm{S}}=45.5 \times 10^{7}$ e.s.u. $/ \mathrm{s}(=0.15 \mathrm{~A})$. For a resistivity $\underline{18} \rho=70 \mu \Omega \mathrm{cm}$ of $2 \mathrm{~nm}$ thick Py at room temperature or resistance $R=0.75 \Omega$ the constant $C_{\mathrm{S}}=17 \mathrm{~mW}$. We consider the data from Fig. 2 for the fidelity time $\tau=1000 \tau_{0}$. For the 'dc' case, the enhancement is achieved by investing $P_{f}=1.8 \times 10^{-2}$ or average power $P=0.31 \mathrm{~m} \mathrm{~W}$. The same order of enhancement in fidelity is obtained by consuming $P_{f}=0.76 \times 10^{-2}$ or $P=0.13 \mathrm{~mW}$ in SFB scheme by applying a current of pulse of equivalent magnetic field $a_{J} / 4 \pi M_{s}=0.275$. For a valve with a cross-section $100 \times 50 \mathrm{~nm}^{2}$ this requires a current of magnitude $I=12.51 \times 10^{7}$ e.s.u. $/ \mathrm{s}(=0.04 \mathrm{~A})$ which corresponds to a current density $8 \times 10^{12} \mathrm{~A} / \mathrm{m}^{2}$. Clearly, at such current densities our approach will break down due to Joule heating. For the IFB scheme the same enhancement in fidelity is achieved by investing $P_{f}=0.13 \times 10^{-2}$ or $P=0.02 \mathrm{~m} \mathrm{~W}$ when $\epsilon=0.5$. This strong reduction in the invested power will alleviate the aforementioned heating problem in SFB case. The efficiency (fidelity) of the operation depends on the different system parameters (spin-torque $a_{J} / I$, external field, anisotropy and demagnetization field etc.), but also on the chosen cut-off value $m_{c}$ and the boundary values $m_{l}$ and $m_{u}$. The qualitative message appears to be valid for all sensible parameter sets, however.

In conclusion, we propose to suppress noise in metallic magnetic spin valves making use of the current-induced spin transfer torque. With moderate power consumption it is possible to increase the fidelity of the device, especially when a feedback loop is implemented which controls the spin-transfer torque depending on the instantaneous magnetization configuration. The latter can be measured by the electric resistance. The correcting current pulses should have sharp rise and decay times which can be generated by state-of-the-art electronics 19 . Our method can be easily adopted to magnetic tunneling junctions. The method can be used to suppress noise in read heads and increase reliability of memory elements.

\section{ACKNOWLEDGMENTS}

This work was supported by the European Union via DYNAMAX and MACALO. SB acknowledges hospitality of TU Delft.

${ }^{1}$ Neil Smith and Patrick Arnett, Appl. Phys. Lett. 78, 1448 (2001). ${ }^{2}$ S. Maat, N. Smith, M. J. Carey, and J. R. Childress, Appl. Phys. Lett. 93, 103506 (2008).

${ }^{3}$ Mark Covington, United States Patent, US 7,042,685 B2(2006).

${ }^{4}$ J. Foros, A. Brataas, Y. Tserkovnyak, and G. E. W. Bauer, Phys. Rev. Lett. 95, 016601 (2005).

${ }^{5}$ J. C. Slonczewski, J. Magn. Magn. Mater. 159, L1 (1996).

${ }^{6}$ L. Berger, Phys. Rev. B 54, 9353 (1996).

${ }^{7}$ D. C. Ralph and M. D. Stiles, J. Magn. Magn. Mater. 247, 1190 (2007).

${ }^{8}$ A. Brataas, Yu. V. Nazarov, and G. E. W. Bauer, Phys. Rev. Lett. 84, 2481 (2000).

${ }^{9}$ A. Brataas, Yu. V. Nazarov, and G. E. W. Bauer, Eur. Phys. J. B 22, 99 (2001).

${ }^{10}$ J. Z. Sun, Phys. Rev. B 62, 570 (2000).

${ }^{11}$ S. I. Kiselev, J. C. Sankey, I. N. Krivorotov, N. C. Emley, A. G. F. Garcia, R. A. Buhrman, and D. C. Ralph, Phys. Rev. B 72, 064430 (2005).

${ }^{12}$ S. Urazhdin, N. O. Birge, W. P. Pratt, Jr., and J. Bass, Phys. Rev. Lett. 91, 146803 (2003).

${ }^{13}$ T. Devolder, J. Hayakawa, K. Ito, H. Takahashi, S. Ikeda, J. A. Katine, M. J. Carey, P. Crozat, J. V. Kim, and C. Chappert, Journal of Appl. Phys. 103, 07A723 (2008).

${ }^{14}$ J. A. Osoborn, Phys. Rev. 67, 351 (1945).

${ }^{15}$ William Fuller Brown, Jr., Phys. Rev. 130, 1677 (1963).

${ }^{16}$ J. L. Garcia-Palacios and F. J. Lazaro, Phys. Rev. B 58, 14937 (1998).

${ }^{17}$ N. C. Emley, I. N. Krivorotov, O. Ozatay, A. G. F. Garcia, J.C. Sankey, D. C. Ralph, and R. A. Buhrman, Phys. Rev. Lett. 96, 247204 (2006).

${ }^{18}$ G. Counil, T. Devolder, J.-V. Kim, P. Crozat1, C. Chappert, S. Zoll, and R. Fournel, IEEE Trans. Mag. 42, 3323 (2006). 
${ }^{19}$ O. J. Lee, V. S. Pribiag, P. M. Braganca, P. G. Gowtham, D. C. Ralph, R. A. Buhrman, and F. J. Lazaro, Appl. Phys. Lett. 95,

012506 (2009). 